\title{
PREFACE Modelling in Ecology, Epidemiology and Evolution
}

\begin{abstract}
A. Morozov*
Abstract. In the preface, we present a short overview of the papers included in the issue of Mathematical Modelling of Natural Phenomena 'Modelling in Ecology, Epidemiology and Evolution'.
\end{abstract}

Mathematics Subject Classification. 35B36, 92C15, 34C23, 34F10, 35B32, 37-xx

Accepted March 26, 2018.

Mathematical Modelling in Ecology, Epidemiology and Evolution is a rapidly growing area of research, which takes advantage of recently developed mathematical and computational techniques as well as novel methods of data analysis. Interestingly, some classical fundamental notions, which have been included into almost all standard student textbooks on mathematical biology (e.g. population fitness, net reproductive number, role of the shape of functional responses in stability of food webs, etc.), are currently being revisited. This special Issue of the Journal is focused on few such topics. In particular, the contributions to the Issue address recent advances in our understanding of the net reproduction function in structured populations, stability of tri-trophic food webs, evolutionary fitness, models of co-infection in epidemiology and economic-demographic models. We should stress, however, that most of the contributions here are not restricted to only one of the mentioned topics but combine several of them, so it may be hard to assign a particular article to a single topic.

In his work [10], Farkas revisits the fundamental notion of the net reproduction function in population models in the case the population is structured. Construction of a net reproduction function in physiologically and agestructured populations is crucial for understanding stability of their stationary states [11-13]. The idea of [10] consists in converting the initial non-linear structured population model into a family of linear ones, where each linear model describes population dynamics in a constant environment. Mathematically, the theory is based on the recently discovered link between the spectral bound of an unbounded operator and the spectral radius of a corresponding bounded operator.

In their work, Zincenko et al. explore possible scenarios of the current growth of the human population across continents which is coupled with dynamics of family wealth and immigration rates [25]. This approach is known as economic-demographic modelling and it is currently increasing its popularity due to the need to explain and predict the observed population decline in some parts of the World such as, for example, Western Europe. The model is described by a system of two ODEs and it is an extension of the previous work by the same co-authors

Keywords and phrases: Economic-demographic model, basic reproduction number, net reproduction function, continuation and bifurcation analysis, chaotic dynamics, evolutionary game models.

Department of Mathematics, University of Leicester, LE1 7RH Leicester, UK.

* Corresponding author: am379@leicester.ac.uk 
[22]. Zincenko et al. predict scenarios of a sharp population decline and deterioration of the economic situation after some periods of intensive transient growth.

The importance of the shape of the functional response in ecological predator-prey models has been previously emphasized $[3,6,15]$. For example, recent studies revealed the role of herd behaviour and collective defence of animals in dynamics of predator-prey systems: animal aggregation of individuals in herds would reduce their consumption by predators and this can be mathematically described via a square root functional response [22]. In their article, Vilches et al. comprehensively explore a predator-prey model with a generic square root functional response [21]. The authors show that the implementation of a square root functional response in the model can result in the extinction of the population within finite time, which can be important for conservation programs.

In their work, Namba et al. [18] address the role of intraguild predation in food webs, which has received less attention in the literature as compared to the classical straight trophic chains [9]. This study considers a tri-trophic ODE-based model and pays a particular attention to the stabilizing role of intra-specific competition between the intermediate and the top predators in the case of a high growth rate of the first trophic level. An important new result of [18] is that in ecosystems with high primary production (eutrophic systems), a strong self-regulation within the population of the intraguild top predator would prevent extinction of the intermediate intraguild predator. This can explain the reported mismatch between empirical observations and theoretical results in ecosystems with intraguild predation [1].

Complicated resource-consumer interaction is considered in [8], where the authors model anaerobic digestion in a microbial ecosystem of a chemostat type. The work explores the syntrophic type of interaction of two microbial species with two substrates, i.e. when the first bacterial species releases a product which is in turn consumed by the other species. This work is an extension of the previous study on the topic by including the realistic assumption about the inhibition of first bacterial species by an excess of the second substrate. With the help of the so-called operating diagrams, the authors list all possible regimes in the system and provide conditions for stabilisation of the non-trivial coexistence equilibria. This study may have practical applications in controlling the production of some biogases (e.g. methane) in industrial anaerobic digestion of bacteria.

In their study, Rodriguez-Rodriguez et al. [19] consider dynamics of structured populations of social insects. Namely, they investigate the interplay between age polyethism, egg cannibalism and division of labour of insects. As an important ecological case study, they explore the dynamics of fungus-gardening leaf-cutter ants which are currently considered to be the greatest agricultural pest of the neotropics. In the paper, it is demonstrated that the age polyethism would play a key role in persistence of the entire colony and that the intermediate strength of cannibalism would be optimal for the survival of the whole population. The findings of the study can help to improve biological control of leaf-cutter ants.

The notion of population fitness and its quantitative description have long been a matter of hot debates in modelling of evolution and adaptation $[4,14,17,24]$. In fact, the initial seminal idea of Wright [24] was to consider each evolutionary process as hill climbing on an adaptive landscape to maximise local fitness. However, this paradigm has been criticized on various backgrounds due to mismatch with both theory and more advanced models. In their work, Bratus et al. [5] try to revisit the original idea of Wright to define generic cases in which adaptive landscape metaphor still holds. They also show that even if fitness is not maximised during evolution, the overall structure of adaptive landscape would still determine the evolutionary dynamics. Mathematically, the study is based on the replicator equation framework.

Finally, the epidemiological part of this special Issue is the study of Marvá [16], which models coinfection of the same organism by two different types of infections: a primary disease and an opportunistic one. This infection scenario is frequently observed in nature. For example, it was reported by the World Health Organization that in the presence of HIV, the rate of developing tuberculosis is much higher than in the case of a healthy individual [23]. In their modelling study, the authors consider the scenario where the opportunistic disease acts on a faster time scale as compared to the primary disease. The basic reproduction numbers in the study are analytically obtained for various scenarios (e.g. for a constant recovery rate and in the case of medical treatment of patients), which overall can be helpful for eventual disease eradication. 


\section{REFERENCES}

[1] P. Amarasekare, Coexistence of intraguild predators and prey in resource-rich environments. Ecology 89 (2008) $2786-2797$.

[2] M. Banerjee, B.W. Kooi and E. Venturino, An ecoepidemic model with prey herd behavior and predator feeding saturation response on both healthy and diseased prey. In Eco-epidemiology. MMNP 12 (2017) 133-161.

[3] A.D. Bazykin, A.I. Khibnik and B. Krauskopf, Nonlinear Dynamics of Interacting Populations. World Scientific (1998).

[4] J. Birch, Natural selection and the maximization of fitness. Biol. Rev. 91 (2016) 712-727.

[5] A.S. Bratus, Y.S. Semenov and A.S. Novozhilov, Adaptive fitness landscape for replicator systems: to maximize or not to maximize. MMNP 13 (2018) 25. DOI: $10.1051 / \mathrm{mmnp} / 2018040$

[6] F. Cordoleani, D. Nerini, M. Gauduchon, A. Morozov and J.C. Poggiale, Structural sensitivity of biological models revisited. J. Theor. Biol. 283 (2011) 82-91.

[7] E. Dangbé, D. Békollé, D. Irépran and A. Perasso, Impact of hygiene, famine and environment on transmission and spread of cholera. In Eco-epidemiology. MMNP 12 (2017) 4-21.

[8] Y. Daoud, N. Abdellatif, T. Sari and J. Harmand, Steady state analysis of a syntrophic association of two species in a chemostat: the effect of a new input concentration substrate. MMNP 13 (2018) 31. DOI: 10.1051/mmnp/2018037

[9] H.I. Egilmez, A.Y. Morozov, Tri-trophic plankton models revised: importance of space, food web structure and functional response parametrisation. In Ecology, Epidemiology and Evolution. MMNP 11 (2016) 16-33.

[10] J. Farkas, Net reproduction functions for nonlinear structured population models. $M M N P \quad \mathbf{1 3}$ (2018) 32. DOI: $10.1051 / \mathrm{mmnp} / 2018036$

[11] J.Z. Farkas and T. Hagen, Asymptotic behavior of size-structured populations via juvenile-adult interaction. Discrete Continuous Dyn. Syst. Ser. B 9 (2008) 249-266.

[12] J.Z. Farkas and A.Y. Morozov, Modelling effects of rapid evolution on persistence and stability in structured predator-prey systems. In Biological evolution. MMNP 9 (2014) 26-46.

[13] J.Z. Farkas, A.Y. Morozov, E.G. Arashkevich and A. Nikishina, Revisiting the stability of spatially heterogeneous predatorprey systems under eutrophication. Bull. Math. Biol. 77 (2015) 1886-1908.

[14] S. Gavrilets, Fitness Landscapes and the Origin of Species. Princeton University Press, Princeton, NJ (2004).

[15] W.C. Gentleman and A.B. Neuheimer, Functional responses and ecosystem dynamics: how clearance rates explain the influence of satiation, food limitation and acclimation. J. Plankt. Res. 30 (2008) 1215-1231.

[16] M. Marvá, R. Bravo de la Parra and E. Venturino, Modelling the role of opportunistic diseases in coinfection. MMNP 13 (2018) 28. DOI: $10.1051 / \mathrm{mmnp} / 2018034$

[17] A.Y. Morozov and O.A. Kuzenkov, Towards developing a general framework for modelling vertical migration in zooplankton. J. Theor. Biol. 405 (2016) 17-28

[18] T. Namba, Y. Takeuchi and M. Banerjee, Stabilizing effect of intra-specific competition on prey-predator dynamics with intraguild predation. MMNP 13 (2018) 29. DOI: 10.1051/mmnp/2018033

[19] M. Rodriguez-Rodriguez, N. Smith, T. Phan, J. Woodbury and Y. Kang, Interactions between leaf-cutter ants and fungus garden: effects of division of labor, age polyethism, and egg cannibalism. MMNP 13 (2018) 30. DOI: 10.1051/mmnp/2018039

[20] E. Venturino. Ecoepidemiology: a more comprehensive view of population interactions. In Reviews in mathematical modelling. MMNP 11 (2016) 49-90.

[21] K. Vilches, E. González-Olivares and A. Rojas-Palma, Prey herd behavior modeled by a generic non-differential functional response. MMNP 13 (2018) 26. DOI: 10.1051/mmnp/2018038

[22] V. Volpert, S. Petrovskii and A. Zincenko, Interaction of human migration and wealth distribution. Nonlinear Anal. 159 (2017) 408-423.

[23] World Health Organization, WHO Report 2011: Global Tuberculosis Control. 2011.

[24] S. Wright, Surfaces of selective value revisited. Am. Nat. 131 (1988) 115-123.

[25] A. Zincenko, S. Petrovskii and V. Volpert, An economic-demographic dynamical system. MMNP 13 (2018) 27. DOI: $10.1051 / \mathrm{mmnp} / 2018038$ 